\title{
Reduction of Commutation Failure Frequency in HVDC Transmission Systems by means of an Improved Solid-State Fault Current Limiter
}

\author{
Sohrab Mirsaeidi \\ School of Electrical Engineering \\ Beijing Jiaotong University \\ Beijing, China \\ msohrab@bjtu.edu.cn \\ Dimitrios Tzelepis \\ Department of Electronic and \\ Electrical Engineering \\ University of Strathclyde \\ Glasgow, UK \\ dimitrios.tzelepis@strath.ac.uk
}

\author{
Hanzhang Liu \\ School of Electrical Engineering \\ Beijing Jiaotong University \\ Beijing, China \\ 16292009@bjtu.edu.cn \\ Dalila Mat Said \\ Faculty of Electrical Engineering \\ Universiti Teknologi Malaysia (UTM) \\ Johor, Malaysia \\ dalila@utm.my
}

\author{
Jinghan $\mathrm{He}$ \\ School of Electrical Engineering \\ Beijing Jiaotong University \\ Beijing, China \\ jhhe@bjtu.edu.cn
}

\begin{abstract}
In the recent decade, High Voltage Direct Current (HVDC) technology has become the preferred option for bulk power transmission due to its numerous advantages such as lower losses and less space requirements compared to High Voltage Alternating Current (HVAC) transmission. However, in order to accelerate the widespread adoption of HVDC systems, some of their technical challenges such as commutation failure need to be resolved. In this study, a Fully Controllable Solid-State Fault Current Limiter (FCSSFCL) is proposed, which is designed based on both fault severity (fault resistance) and fault initiation time. It inserts an adequate number of resistors in the fault current path so that the commutation failure is prevented. The salient feature of the proposed FCSSFCL is that it is fully controllable, and hence it prevents additional power losses in the inverter AC system. The importance of the proposed FCSSFCL becomes more evident in a power system, wherein the inverter supplies more than one AC system. In such a network, insertion of a large resistance by the FCL during a non-severe fault at one of the inverter AC systems leads to the unnecessary reduction of power flow in other non-faulted AC systems. In order to validate the efficacy of the proposed FCSSFCL, several simulations have been performed under different fault types. The simulation results indicate that the proposed approach can effectively reduce the commutation failure frequency.
\end{abstract}

Keywords-HVDC transmission, line-commutated converters, commutation failure, solid-state fault current limiters.

\section{INTRODUCTION}

Nowadays, HVDC technology is considered as a suitable alternative for bulk power transmission over long distances. It offers several advantages in comparison with traditional HVAC system such as asynchronous interconnection, reduction in line losses, and improvement of network stability [1]. Line-Commutated Converters (LCC) and Voltage Source Converters (VSC) are two dominant types of HVDC converters [2]. Even though the VSC is a more modern technology and is used for more diverse applications, the efficiency and transmission capability of LCCs are still higher than VSCs. In VSCs, Insulated gate bipolar transistor (IGBT) switches are used which have superior controllability, while the switching of LCCs is performed by thyristors with only turn-off capability. Therefore, LCCs are vulnerable to commutation failure which is mainly caused by the voltage drop at the inverter AC system [3].

Commutation failure is an adverse dynamic event which may lead to some serious transient phenomena such as temporary interruption of HVDC transmission power, increased stress on the network equipment, overheating of the converter valves, and malfunction of the protection system. If commutation failure is not inhibited and continuously repeats, the converter is forcibly blocked which leads to a large-scale blackout at the inverter AC system [4]. As a result, to propose a countermeasure for effective commutation failure prevention is of great importance. The existing commutation failure strategies can be categorized into two groups, i.e. improvement of HVDC control system performance, and addition of extra devices [5].

For the first group, the main idea is to swiftly detect the inverter AC fault and decrease the firing angle order applied to the inverter thyristors so that the commutation margin becomes larger and the commutation failure is prevented. In references [6]-[14], various strategies have been proposed which are different in terms of fault detection type, accuracy in calculation of the reduced commutation margin, and the speed of response to commutation failure. Nevertheless, such strategies are not appropriate options for dealing with severe commutation failures. In fact, the main purpose of these approaches is to reduce the number or impact of commutation failures.

In order to remedy the challenges of strategies based on improvement of HVDC control system, the methods in the second group propose installation of extra devices at the inverter side. In [15], [16], adoption of Superconducting Fault Current Limiter (SFCL) is suggested as an effective way for commutation failure prevention. The function of SFCLs is to inhibit the excessive voltage drop at the inverter $\mathrm{AC}$ bus through inserting a large impedance in the fault current path. However, the main drawback of the SFCLs is that they are not able to insert a desired 
impedance, since they function based on a quenching characteristic, wherein the current level at which the quench occurs is dependent on the operating temperature, and the amount and type of superconductor.

In this paper, based on further research and simulation, a Fully Controllable Superconducting Fault Current Limiter (FCSSFCL) is proposed which prevents the commutation failure by inserting a suitable resistance in the fault current path through a multi-switch circuit. The main advantage of the presented FCSSFCL is that the number of inserted resistances can be selected according to the severity of the fault which prevents the additional power losses in the inverter AC system.

The remainder of this paper is organized as follows: In Section II, the mechanism of commutation failure is introduced; in Section III, the proposed commutation failure prevention approach is presented; Section IV discusses the obtained simulation results; and finally, Section V concludes the paper.

\section{MECHANISM OF COMMUTATION FAILURE}

The most frequenctly used structure for linecommutated converters is shown in Fig. 1 which is referred to as Graetz bridge. As can be seen from the figure, it includes six thyristor valves which are numbered based on their firing sequence. Since each valve conducts for 120 degrees, such structure is termed as 6-pulse converter. Line commutated converters can be used for transmission of power from AC to DC side (rectification) or from DC to AC side (inversion) by applying different firing angles to the valves. As mentioned before, thyristor switches have no turn-off capability. As a result, for successful turn-off of these valves, a negative anode-cathode voltage needs to be applied across the valves which is provided by the inverter AC voltage. When a fault happens at the inverter AC side, a voltage drop appears at the inverter bus and the switching valves cannot be turned off. Such a phenomenon is termed as commutation failure which is a frequent event in linecommutated HVDC systems.

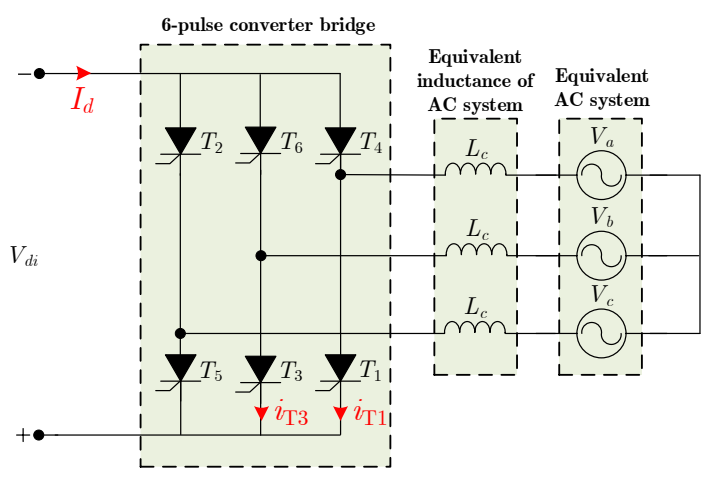

Fig. 1. Structure of a 6-pulse line-commutated converter.

Fig. 2 illustrates the commutation process between valves $T_{1}$ and $T_{3}$ of the converter shown in Fig.1. As can be seen from the figure, the conduction of the valve $T_{1}$ cannot be transferred to valve $T_{3}$ instantaneously due to the equivalent inductance of the inverter AC system $L_{\mathrm{c}}$ and takes for a certain time which corresponds to the overlap area $A_{\mu}$. The voltage equation for the loop including valves $T_{1}$ and $T_{3}$ can be written as:

$$
\overbrace{\sqrt{2} V_{L L} \sin \omega t}^{V_{b a}}=L_{c} \omega \frac{d i_{T 3}}{d \omega t}-L_{c} \omega \frac{d \overbrace{\frac{\left(I_{d-}-i_{T 3}\right)}{d \omega t}}^{i_{T 1}}}{d \omega}
$$

where $V_{b a}$ is the voltage across valves $T_{1}$ and $T_{3}$ which is termed as commutation voltage. In this equation, since the DC current is constant, $d I_{d} / d \omega t=0$. Integrating from both sides of (1) results in:

$$
\overbrace{\int_{\alpha_{i}}^{\pi-\gamma_{i}} \sqrt{2} V_{L L} \sin \omega t}^{A_{\mu i}}=2 \omega L_{c} \overbrace{\int_{0}^{I_{d}} d i_{T 3}}^{I_{d}}
$$

From this equation, it can be concluded that:

$$
A_{\mu i} \propto I_{d}
$$

In case a fault occurs at the inverter $\mathrm{AC}$ side, the commutating voltage of the affected phases will decrease in magnitude. For example, in case a symmetrical threephase fault takes place, the magnitude of all phase voltages decreases, and hence a voltage drop happens on commutation voltage $V_{b a}$. Such an AC voltage reduction leads to the decrease of the DC voltage. However, since the transmitted power in an HVDC system is controlled to be constant, the DC voltage drop leads to the sudden increase of the DC current which is proportional to $A_{\mu i}$ according to (3). Therefore, according to Fig. 2, both decrease of $V_{b a}$ and increase of $A_{\mu i}$ will result in a severe reduction of commutation margin $\left(A_{\gamma i}\right)$. Consequently, valve $T_{1}$ will not be able to receive adequate negative anode-cathode voltage, and hence cannot be turned off. In the next switching sequence, $T_{4}$ also turns on. This means that both valves $T_{1}$ and $T_{4}$ which belong to one converter valve conduct at the same time and a short circuit occurs on the HVDC line. If such a phenomenon repeats several times, the converter is shut down after about $500 \mathrm{~ms}$ which results in a large blackout at the inverter AC system.

It should be stressed that since the commutation margin in the inverter side is much smaller than the rectifier side, the risk of commutation failure for the inverter is much higher. As a result, the majority of commutation failure strategies are designed for the inverter side.

In addition to AC faults, an internal malfunction of the converter firing control system or an action of the protection function may lead to commutation failure. In such circumstances, no firing pulses are applied to the valves, and hence no power is transmitted from DC to AC side. However, in this research, only commutation failures caused by AC faults are investigated.

\section{Proposed Commutation FAILURE PREVENTION APPROACH}

\section{A. Design of the Proposed FCSSFCL}

Fig. 3 depicts the flow chart for the design of the proposed FCSSFCL. As mentioned before, fault current limiters can effectively inhibit commutation failure through inserting suitable impedance in the fault current path. When a fault occurs at the inverter AC system, the inverter AC voltage is reduced depending on the fault severity and the fault initiation time. Also, since the fault leads to the enlargement of $A_{\mu i}$, the DC current increases. Therefore, commutation failure phenomenon can be recognized through changes of both AC voltage and DC current. However, since the speed of response is one of the most important factors in the commutation failure elimination 


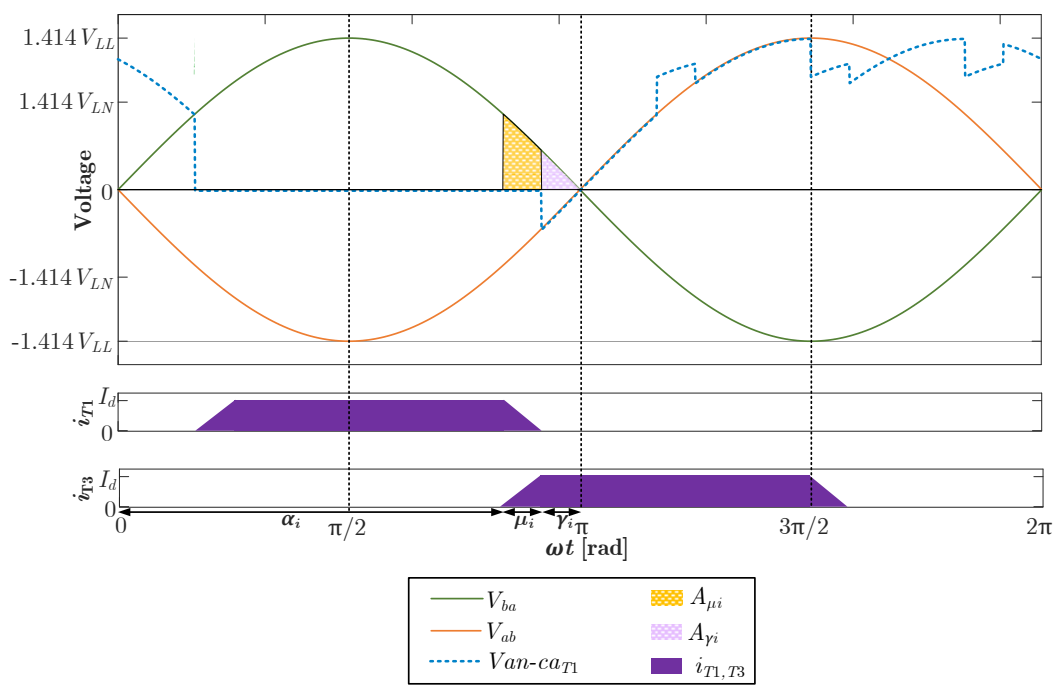

Fig. 2. Commutation process between valves $T_{1}$ and $T_{3}$ of the converter shown in Fig.1

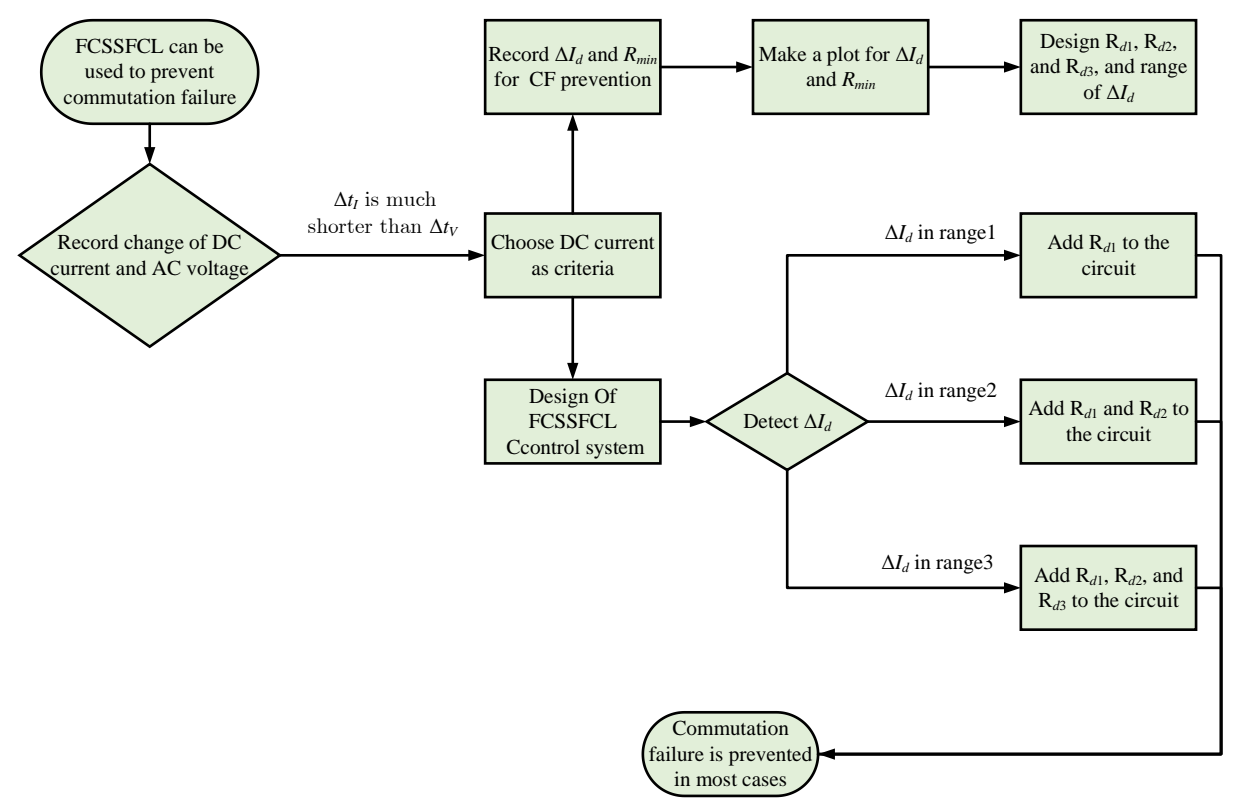

Fig. 3. Flow chart for the design of the proposed FCSSFCL.

TABLE I. DIFFERENCE BETWEEN CHANGE RATE OF THE DC CURRENT AND AC VOLTAGE DURING A TYPICAL INVERTER AC FAULT FOR FIVE VARIOUS FAULT INITIATION TIMES

\begin{tabular}{|c|c|c|c|c|c|}
\hline \multirow{2}{*}{$\boldsymbol{R}_{f}[\mathbf{\Omega}]$} & \multicolumn{5}{|c|}{ Fault Initiation Time [s] } \\
\hline & 1.000 & 1.002 & 1.004 & 1.006 & 1.008 \\
\hline \multirow[t]{4}{*}{75} & \multicolumn{5}{|c|}{$\Delta V_{a c}=10.72 \mathrm{kV}$} \\
\hline & \multicolumn{5}{|c|}{$\Delta t_{V}=0.032 \mathrm{~s}$} \\
\hline & \multicolumn{5}{|c|}{$\Delta I_{d}=0.38 \mathrm{kA}$} \\
\hline & \multicolumn{5}{|c|}{$\Delta t_{I}=0.009 \mathrm{~s}$} \\
\hline \multirow[t]{4}{*}{65} & & $\Delta V_{a c}=9.24 \mathrm{kV}$ & $\Delta V_{a c}=14.43 \mathrm{kV}$ & & \\
\hline & & $\Delta t_{V}=0.034 \mathrm{~s}$ & $\Delta t_{V}=0.034 \mathrm{~s}$ & & \\
\hline & & $\Delta I_{d}=0.34 \mathrm{kA}$ & $\Delta I_{d}=0.50 \mathrm{kA}$ & & \\
\hline & & $\Delta t_{I}=0.011 \mathrm{~s}$ & $\Delta t_{I}=0.010 \mathrm{~s}$ & & \\
\hline \multirow[t]{4}{*}{55} & $\Delta V_{a c}=10.45 \mathrm{kV}$ & $\Delta V_{a c}=12.90 \mathrm{kV}$ & $\Delta V_{a c}=18.52 \mathrm{kV}$ & $\Delta V_{a c}=10.62 \mathrm{kV}$ & \\
\hline & $\Delta t_{V}=0.036 \mathrm{~s}$ & $\Delta t_{V}=0.034 \mathrm{~s}$ & $\Delta t_{V}=0.031 \mathrm{~s}$ & $\Delta t_{V}=0.030 \mathrm{~s}$ & \\
\hline & $\Delta I_{d}=0.35 \mathrm{kA}$ & $\Delta I_{d}=0.46 \mathrm{kA}$ & $\Delta I_{d}=1.90 \mathrm{kA}$ & $\Delta I_{d}=0.37 \mathrm{kA}$ & \\
\hline & $\Delta t_{I}=0.015 \mathrm{~s}$ & $\Delta t_{I}=0.009 \mathrm{~s}$ & $\Delta t_{I}=0.010 \mathrm{~s}$ & $\Delta t_{I}=0.008 \mathrm{~s}$ & \\
\hline \multirow[t]{4}{*}{45} & $\Delta V_{a c}=13.63 \mathrm{kV}$ & $\Delta V_{a c}=19.00 \mathrm{kV}$ & $\Delta V_{a c}=19.36 \mathrm{kV}$ & $\Delta V_{a c}=13.33 \mathrm{kV}$ & \\
\hline & $\Delta t_{V}=0.036 \mathrm{~s}$ & $\Delta t_{V}=0.029 \mathrm{~s}$ & $\Delta t_{V}=0.031 \mathrm{~s}$ & $\Delta t_{V}=0.030 \mathrm{~s}$ & \\
\hline & $\Delta I_{d}=0.46 \mathrm{kA}$ & $\Delta I_{d}=1.88 \mathrm{kA}$ & $\Delta I_{d}=1.90 \mathrm{kA}$ & $\Delta I_{d}=0.45 \mathrm{kA}$ & \\
\hline & $\Delta t_{I}=0.014 \mathrm{~s}$ & $\Delta t_{I}=0.009 \mathrm{~s}$ & $\Delta t_{I}=0.010 \mathrm{~s}$ & $\Delta t_{I}=0.008 \mathrm{~s}$ & \\
\hline \multirow[t]{4}{*}{35} & $\Delta V_{a c}=19.70 \mathrm{kV}$ & $\Delta V_{a c}=20.46 \mathrm{kV}$ & $\Delta V_{a c}=20.26 \mathrm{kV}$ & $\Delta V_{a c}=22.70 \mathrm{kV}$ & $\Delta V_{a c}=13.02 \mathrm{kV}$ \\
\hline & $\Delta t_{V}=0.030 \mathrm{~s}$ & $\Delta t_{V}=0.029 \mathrm{~s}$ & $\Delta t_{V}=0.031 \mathrm{~s}$ & $\Delta t_{V}=0.030 \mathrm{~s}$ & $\Delta t_{V}=0.039 \mathrm{~s}$ \\
\hline & $\Delta I_{d}=1.93 \mathrm{kA}$ & $\Delta I_{d}=1.92 \mathrm{kA}$ & $\Delta I_{d}=1.98 \mathrm{kA}$ & $\Delta I_{d}=1.94 \mathrm{kA}$ & $\Delta I_{d}=0.44 \mathrm{kA}$ \\
\hline & $\Delta t_{I}=0.014 \mathrm{~s}$ & $\Delta t_{I}=0.011 \mathrm{~s}$ & $\Delta t_{I}=0.011 \mathrm{~s}$ & $\Delta t_{I}=0.010 \mathrm{~s}$ & $\Delta t_{I}=0.015 \mathrm{~s}$ \\
\hline
\end{tabular}


strategies, in this study only DC current is used which has a higher change rate compared to the $\mathrm{AC}$ voltage.

The difference between change rate of the DC current and $\mathrm{AC}$ voltage during a typical inverter $\mathrm{AC}$ fault for five various fault initiation times is shown in Table I. In this table, $\Delta I_{d}$ is the difference between the maximum value of $I_{d}$ after a commutation failure and the value of $I_{d}$ in the normal conditions, and $\Delta t_{I}$ is the corresponding time to reach this maximum value. Similarly, $\Delta V_{a c}$ and $\Delta t_{V}$ are used to show the rate of $\mathrm{AC}$ voltage changes. In order to design a controllable resistance for the FCSSFCL, the first step is to find the relationship between DC current changes and the minimum resistance required for prevention of commutation failure. Fig. 4 indicates the simulation results for the determination of minimum FCSSFCL resistance $R_{\text {min }}$ which prevents the commutation failure under different fault resistances and fault initiation times. The obtained data from Fig. 4 can be classified into three ranges, as shown in Table II.

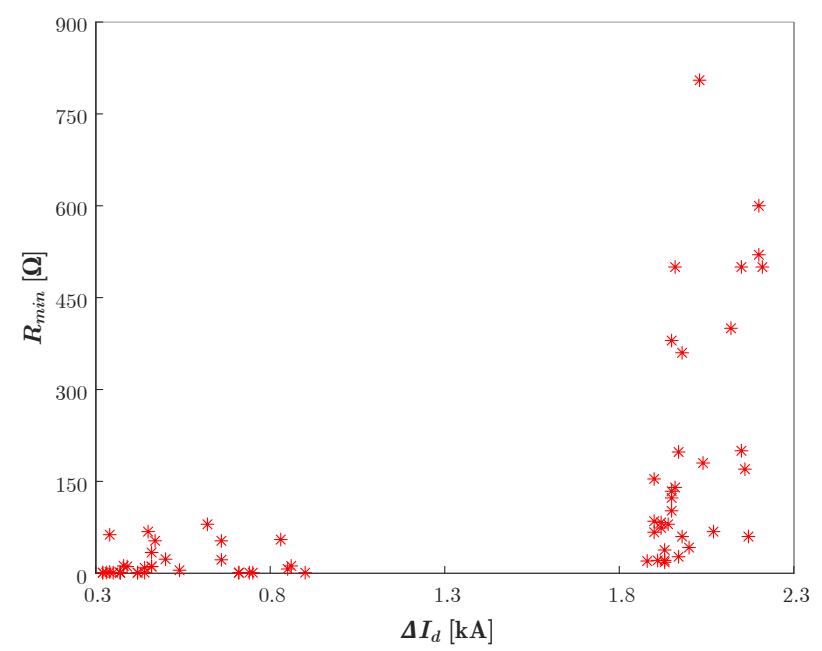

Fig. 4. Simulation results for the determination of minimum FCSSFCL resistance which prevents the commutation failure under different fault resistances and fault initiation times.

TABLE II. CLASSIFICATION OF THE OBTAINED DATA FROM FIG. 4

\begin{tabular}{cccc}
\hline Ranges & $\begin{array}{c}\text { Range of } \\
\boldsymbol{I}_{\boldsymbol{d}}[\mathbf{k A}]\end{array}$ & $\begin{array}{c}\text { Range of } \\
\boldsymbol{R}_{\boldsymbol{m i n}}[\boldsymbol{\Omega}]\end{array}$ & $\begin{array}{c}\text { Number } \\
\text { of cases }\end{array}$ \\
\hline Range1 & $0.32-0.9$ & $1-80$ & 33 \\
Range2 & $1.88-2.01$ & $18-50$ & 22 \\
Range3 & $2.02-2.21$ & $60-805$ & 11 \\
\hline
\end{tabular}

\section{B. Structure of the Proposed FCSSFCL}

As discussed in Section I, the main demerit of SFCLs is that they are not able to insert a desired impedance, since they function based on a quenching characteristic, wherein the current level at which the quench occurs is dependent on the operating temperature, and the amount and type of superconductor. To obviate this challenge, in this study, a Fully Controllable Solid-State Fault Current Limiter (FCSSFCL) is proposed which the process of its design was explained in Section III.A. Fig. 5 demonstrates the schematic diagram of the proposed FCSSFCL in a hybrid AC/DC grid which includes two receiving AC systems. The five main parts of the proposed FCSSFCL are as follows:

1) A three-phase transformer with the turns ratio of $\alpha$ in series with the receiving $\mathrm{AC}$ system. The main purpose of using this transformer is to isolate the FCSSFCL from the main network and reduce the current at a suitable level for the power electronic devices.

2) A three-phase diode bridge rectifier.

3) Three series modules, each of which contains a Self Turn-off Switch (STS) in parallel with a resistor, $R_{d}$. These modules are used for limiting the fault current under different ranges.

4) FCSSFCL control system, which is the most important part of the strategy. It determines the number of resistors required to be included in the circuit by applying suitable firing pulses to the STSs.

5) A small reactor $\left(L_{d}\right)$ which is required to prevent severe $d i / d t$ on the STS. Since the value of $L_{d}$ is small, it is usually equipped with air core to prevent its saturation.

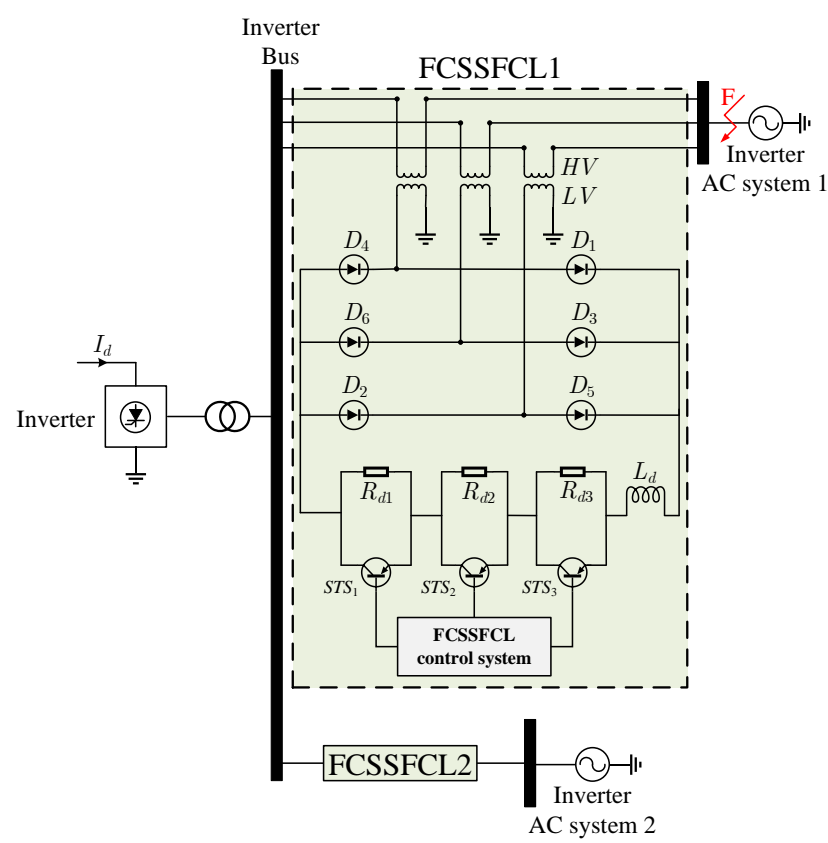

Fig. 5. Schematic diagram of the proposed FCSSFCL.

Table III shows the suitable ranges of $\Delta I_{d}$ and the value of resistors $R_{d 1}, R_{d 2}$, and $R_{d 3}$ for the design of the FCSSFCL which are determined according to the obtained data in Table II.

TABLE III. SUITABLE RANGES OF $\Delta I_{d}$ AND RESISTANCES FOR THE DESIGN OF FCSSFCL.

\begin{tabular}{|c|c|c|}
\hline $\boldsymbol{R}_{\boldsymbol{d}}$ & Range of $\Delta I_{d} \quad[\mathrm{kA}]$ & Resistance [ $\Omega$ ] \\
\hline$R_{d 1}$ & $0.3-0.9$ & 80 \\
\hline$R_{d 2}$ & $1.88-2.01$ & 420 \\
\hline$R_{d 3}$ & more than 2.02 & 305 \\
\hline
\end{tabular}

During the normal operation of the FCSSFCL, all STSs are on, and hence $R_{d 1}, \mathrm{R}_{\mathrm{d} 2}$, and $\mathrm{R}_{\mathrm{d} 3}$ are all bypassed. As a result, the small reactor $L_{d}$ is fully charged and acts as a short-circuit. If the small voltage drop on the semiconductor device is ignored, then the total voltage across the diode bridge is approximately zero. Therefore, the impact of existence of the proposed FCSSFCL can be neglected under normal conditions. 


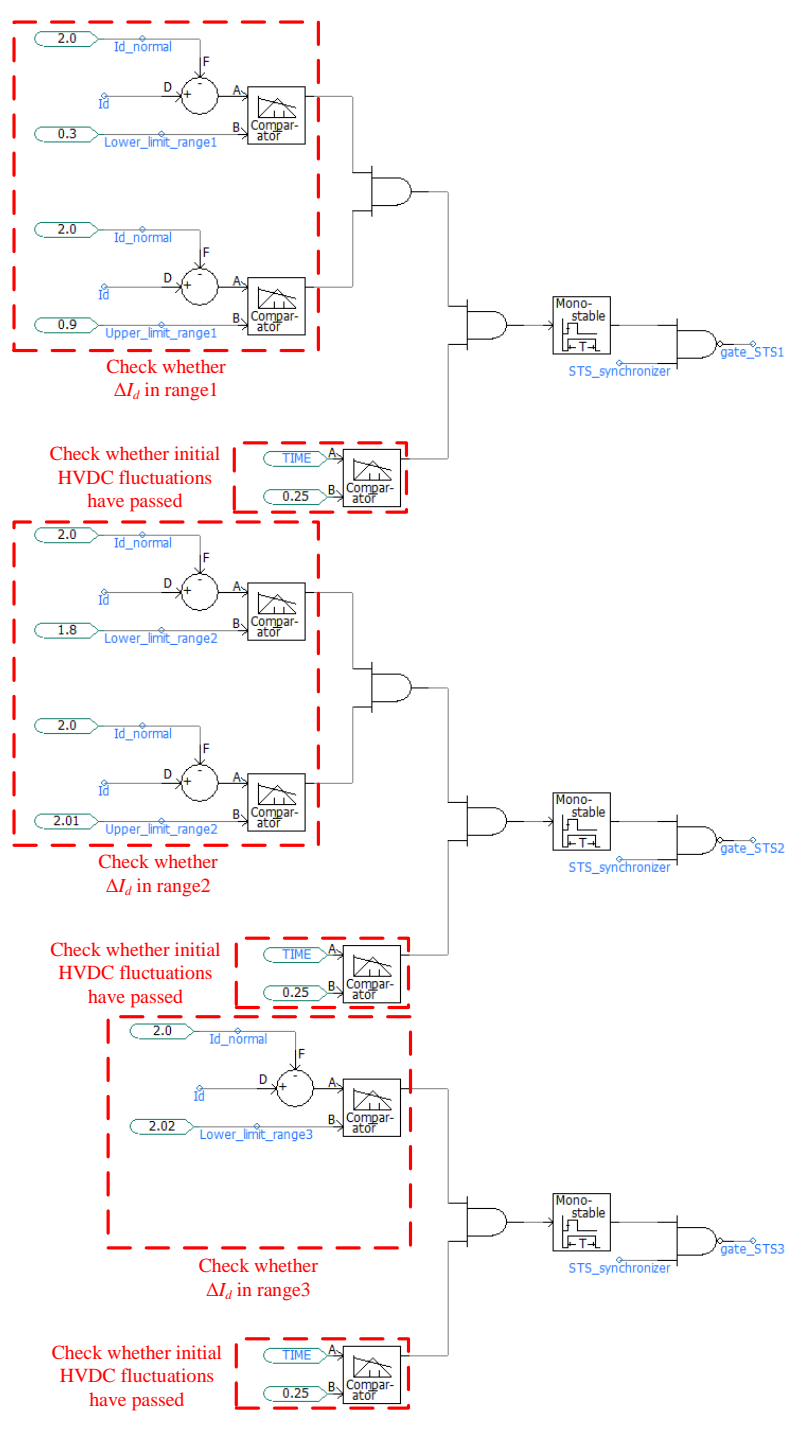

Fig. 6. Structure of the FCSSFCL control system.

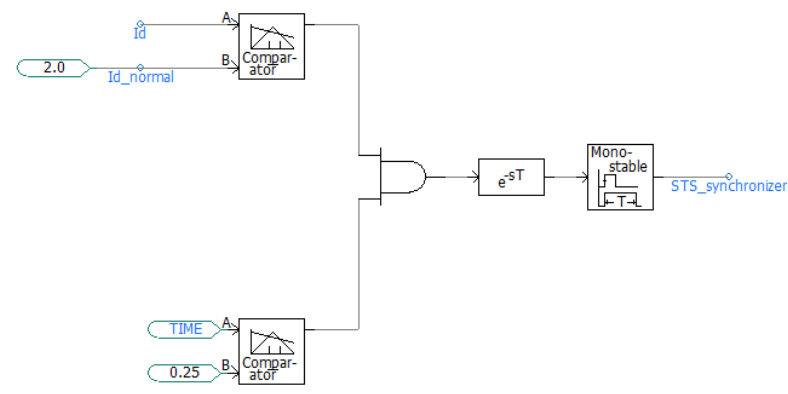

Fig. 7. Structure of STS synchronizer.

When a fault occurs at point F, FCSSFCL control system detects it through measuring the difference between pre-fault and fault currents, $\Delta I_{d}$. Subsequently, it inserts the adequate number of resistors according to the measured value of $\Delta I_{d}$, so that the commutation failure is inhibited.

The structure of the FCSSFCL control system is shown in Fig. 6. As can be seen from the figure, if $\Delta I_{d}$ is between 0.3 and $0.9 \mathrm{kA}$, a pulse will be delivered to the gate of $S T S_{1}$ and only $R_{d 1}$ is inserted into the circuit. However, if $\Delta I_{d}$ is in the range of 1.8 to $2.01 \mathrm{kA}$, the gates of both
$S T S_{1}$ and $S T S_{2}$ receive a pulse signal and both $R_{d 1}$ and $R_{d 2}$ are inserted. In case $\Delta I_{d}$ is greater than $2.02 \mathrm{kA}$, then all switches are triggered, and hence all of the three resistors are put in the FCSSFCL circuit. Also, in order to prevent the malfunction of the FCSSFCL during the initial HVDC fluctuations, a minimum activation time is considered. The FCSSFCL control system also includes an STS synchronizer which is used for the cases, in which more than one resistor is required. Fig. 7 shows the structure of the STS synchronizer. The function of STS synchronizer is to coordinate the firing pulses applied to the switches so that they are triggered at the same time. It shall be noted that for the design of the control system, a monostable multivibrator is also required which its task is to create a pulse with a desirable duration once it receives a trigger signal.

\section{Simulation RESULtS}

In order to validate the effectiveness of the proposed FCSSFCL, several simulations have been conducted using PSCAD/EMTDC software. The single-line of the test network is shown in Fig. 8. As can be seen, it includes a mono-polar $500 \mathrm{kV}$ and $1000 \mathrm{MW}$ HVDC link with 12pulse converters on both rectifier and inverter sides. The test network also includes shunt capacitors, smoothing reactors, low-pass and high-pass filters in both sides of the HVDC line. The detailed parameters of the test network are listed in Table IV.

TABLE IV. PARAMETERS OF THE TEST NetWork

\begin{tabular}{ccc}
\hline Parameter [Unit] & Rectifier & Inverter \\
\hline AC system frequency $[\mathrm{Hz}]$ & 50 & 50 \\
Bus line-to-line voltage $[\mathrm{kV}]$ & 345 & 230 \\
Nominal firing angle $[\mathrm{deg}]$ & 5 & - \\
Nominal extinction angle $[\mathrm{deg}]$ & - & 15 \\
Turn-off time of thyristor $[\mu \mathrm{s}]$ & 30 & 30 \\
\hline
\end{tabular}

Because of the fact that the AC voltage is sinusoidal, the performance of the proposed FCSSFCL does not only depend on the fault type and fault resistance, but also on the fault initiation time. The results obtained from the simulation of various types of faults are indicated in Tables $\mathrm{V}$ to VIII. In each of the tables, the effectiveness of the proposed FCSSFCL for ten fault initiation times ranged from 1 to $1.018 \mathrm{~s}$ is evaluated. The results are reported from the highest fault impedance which leads to the commutation failure. In Tables V to VIII, white cells denote the cases, in which no failed commutation is observed even without applying the FCSSFCL. Also, the gray cells show the cases, in which the inverter is faced with commutation failure, but the FCSSFCL can effectively prevent it. However, the cells which are specified with black color denote the cases, wherein commutation failure occurs but the proposed FCSSFCL is not able to prevent it even by inserting all of the resistors in the circuit. With regard to the obtained results, it can be concluded that the proposed strategy is highly effective in most of the simulation cases.

\section{CONCLUSION}

In this paper, a fully controllable solid-state fault current limiter for the prevention of commutation failure in LCC-HVDC system is proposed. The proposed FCSSFCL 


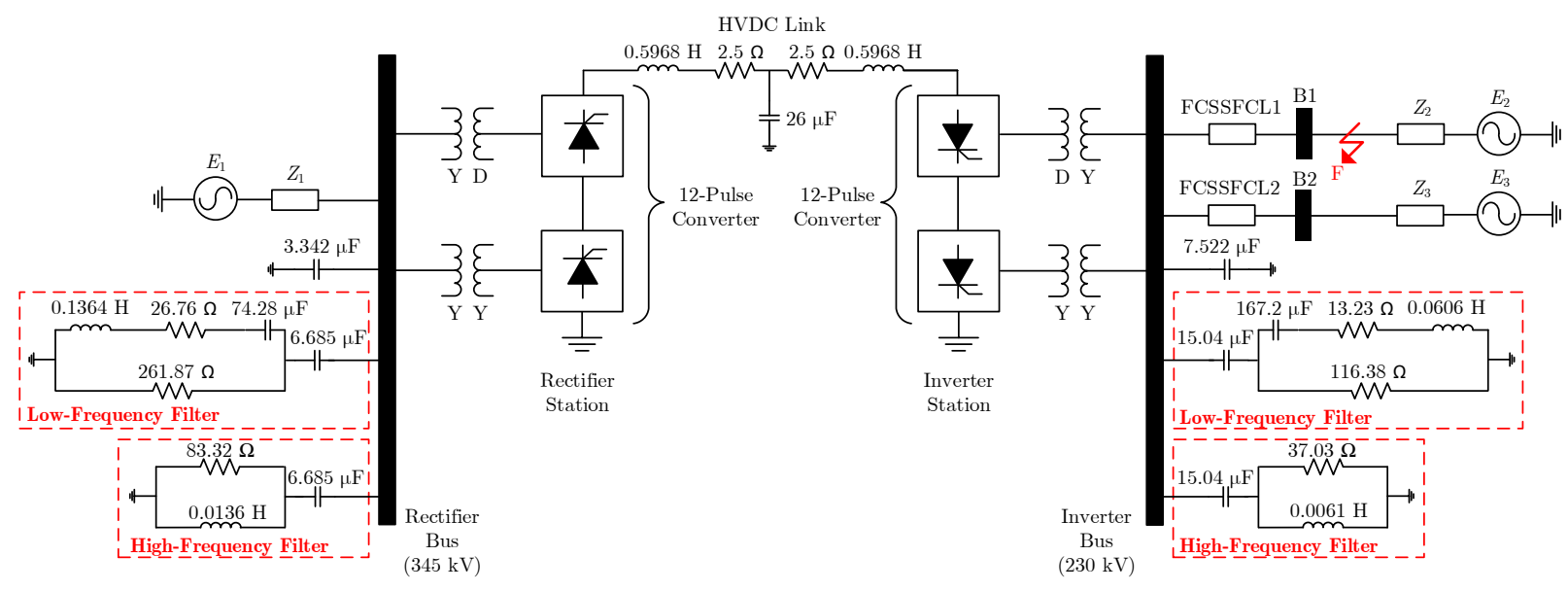

Fig. 8. Single-line of the test network.

TABLE V. IMPROVEMENT OF COMMUTATION FAILURE PREVENTION DURING SINGLE-LINE-TO-GROUND FAULT

\begin{tabular}{|c|c|c|c|c|c|c|c|c|c|c|c|}
\hline \multirow{2}{*}{$\boldsymbol{R}_{f}[\boldsymbol{\Omega}]$} & \multicolumn{7}{|c|}{ Fault Initiation Time [s] } & \multicolumn{7}{c|}{} \\
\hline & 1.000 & 1.002 & 1.004 & 1.006 & 1.008 & 1.010 & 1.012 & 1.014 & 1.016 & 1.018 \\
\hline 95 & & & & & & & & & & \\
\hline 85 & & & & & & & & & & \\
\hline 75 & & & & & & & & & & \\
\hline 65 & & & & & & & & & & \\
\hline 55 & & & & & & & & & & \\
\hline 45 & & & & & & & & & & \\
\hline 35 & & & & & & & & & & \\
\hline 25 & & & & & & & & & & \\
\hline$R_{f}<15$
\end{tabular}

TABLE VI. IMPROVEMENT OF COMMUTATION FAILURE PREVENTION DURING DOUBLE-LINE FAULT

\begin{tabular}{|c|c|c|c|c|c|c|c|c|c|c|}
\hline \multirow{2}{*}{$\boldsymbol{R}_{f}[\boldsymbol{\Omega}]$} & \multicolumn{10}{|c|}{ Fault Initiation Time [s] } \\
\hline & 1.000 & 1.002 & 1.004 & 1.006 & 1.008 & 1.010 & 1.012 & 1.014 & 1.016 & 1.018 \\
\hline \multicolumn{11}{|l|}{365} \\
\hline \multicolumn{11}{|l|}{315} \\
\hline \multicolumn{11}{|l|}{265} \\
\hline \multicolumn{11}{|l|}{215} \\
\hline \multicolumn{11}{|l|}{165} \\
\hline \multicolumn{11}{|l|}{115} \\
\hline$R_{f}<65$ & & & & & & & & & & \\
\hline
\end{tabular}

TABLE VII. IMPROVEMENT OF COMMUTATION FAILURE PREVENTION DURING DOUBLE-LINE-TO-GROUND FAULT

\begin{tabular}{|c|c|c|c|c|c|c|c|c|c|c|c|}
\hline \multirow{2}{*}{$\boldsymbol{R}_{f}[\Omega]$} & \multicolumn{9}{|c|}{ Fault Initiation Time [s] } & \multicolumn{7}{c|}{} \\
\hline & 1.000 & 1.002 & 1.004 & 1.006 & 1.008 & 1.010 & 1.012 & 1.014 & 1.016 & 1.018 \\
\hline 130 & & & & & & & & & & \\
\hline 115 & & & & & & & & & & \\
\hline 100 & & & & & & & & & & \\
\hline 85 & & & & & & & & & & \\
\hline 70 & & & & & & & & & & \\
\hline 55 & & & & & & & & & & \\
\hline 40 & & & & & & & & & & \\
\hline 25 & & & & & & & & & \\
\hline$R_{f}<10$
\end{tabular}


TABLE VIII. IMPROVEMENT OF COMMUTATION FAILURE PREVENTION DURING THREE-PHASE FAULT

\begin{tabular}{|c|c|c|c|c|c|c|c|c|c|c|c|}
\hline \multirow{2}{*}{$\boldsymbol{R}_{f}[\mathbf{\Omega}]$} & \multicolumn{7}{|c|}{ Fault Initiation Time [s] } & \multicolumn{7}{c|}{} \\
\hline & 1.000 & 1.002 & 1.004 & 1.006 & 1.008 & 1.010 & 1.012 & 1.014 & 1.016 & 1.018 \\
\hline 135 & & & & & & & & & & \\
\hline 120 & & & & & & & & & & \\
\hline 105 & & & & & & & & & & \\
\hline 90 & & & & & & & & & & \\
\hline 75 & & & & & & & & & & \\
\hline$R_{f}<60$ & & & & & & & & & \\
\hline
\end{tabular}

detects the inverter AC fault occurrence through measuring the difference between pre-fault and fault currents, and adds an adequate number of resistors to the circuit to prevent commutation failure. Compared to other commutation failure inhibition techniques, the proposed FCSSFCL is fully controllable, and hence it prevents additional power losses in the inverter AC system. To verify the effectiveness of the proposed approach, several simulations have been undertaken using PSCAD/EMTDC software. The obtained results indicate that the proposed FCSSFCL is able to effectively prevent commutation failure and increase the reliability of the inverter AC system.

\section{ACKNOWLEDGMENT}

This work was supported in part by the National Key Research and Development Plan of China (2018YFB0904602), and in part by the Fundamental Research Funds for the Central Universities (2019RC051).

\section{REFERENCES}

[1] B. Hu, K. Xie, and H. M. Tai, "Optimal reliability allocation of 800 $\mathrm{kV}$ ultra HVDC transmission systems," IEEE Trans.PowerDel., vol. 33, no. 3, pp. 1174-1184, Jun. 2018.

[2] Y. Ma, H. Li, G. Wang, and J. Wu, "Fault analysis and travelingwave-based protection scheme for double-circuit LCC-HVDC transmission lines with shared towers, "IEEE Trans. Power Del., vol. 33, no. 3, pp. 1479-1488, Jun. 2018.

[3] S. F. Huang, H. M. Shen, B. Fei, and O. Li, "Effect of commutation failure on the distance protection and the countermeasures," IET Gener., Transmiss. Distribution, vol. 9, no. 9, pp. 838-844, Jun. 2015.

[4] Y. Xue, X. P. Zhang, and C. Yang, "AC filterless flexible LCC HVDC with reduced voltage rating of controllable capacitors," IEEE Trans. Power Syst., vol. 33, no. 5, pp. 5507-5518, Sep. 2018.

[5] J. Chen, Q. Wang, and X. Zhu, "Evaluation of commutation failure risk for HVDC caused by harmonic voltage," in 2019 IEEE 3rd Conference on Energy Internet and Energy System Integration (EI2), 2019, pp. 248-252.

[6] S. Tamai, H. Naitoh, F. Ishiguro, M. Sato, K. Yamaji, and N. Honjo, "Fast and predictive HVDC extinction angle control," IEEE Transactions on Power Systems, vol. 12, no. 3, pp. 1268-1275, Aug 1997.

[7] C. V. Thio, J. B. Davies, and K. L. Kent, "Commutation failures in HVDC transmission systems," IEEE Transactions on Power Delivery, vol. 11, no. 2, pp. 946-957, Apr 1996.

[8] S. Mirsaeidi and X. Dong, "An enhanced strategy to inhibit commutation failure in line-commutated converters," IEEE Transactions on Industrial Electronics, vol. 67, no. 1, pp. 340-349, Jan 2020.

[9] C. Guo, Y. Liu, C. Zhao, X. Wei, and W. Xu, "Power component fault detection method and improved current order limiter control for commutation failure mitigation in HVDC," IEEE Transactions on Power Delivery, vol. 30, no. 3, pp. 1585-1593, June 2015.

[10] J. Bauman and M. Kazerani, "Commutation failure reduction in HVDC systems using adaptive fuzzy logic controller," IEEE Transactions on Power Systems, vol. 22, no. 4, pp. 1995-2002, Nov 2007.
[11] Y. Z. Sun, L. Peng, F. Ma, G. J. Li, and P. F. Lv, "Design a fuzzy controller to minimize the effect of HVDC commutation failure on power system," IEEE Transactions on Power Systems, vol. 23, no. 1, pp. 100-107, Feb 2008.

[12] A. Hansen and H. Havemann, "Decreasing the commutation failure frequency in HVDC transmission systems, " IEEE Transactions on Power Delivery, vol. 15, no. 3, pp. 1022-1026, July 2000.

[13] S. Mirsaeidi, X. Dong, D. Tzelepis, D. M. Said, A. Dysko, and C. Booth, "A predictive control strategy for mitigation of commutation failure in LCC-based HVDC systems," IEEE Transactions on Power Electronics, vol. 34, no. 1, pp. 160-172, Jan 2019.

[14] W. Yao, C. Liu, J. Fang, X. Ai, J. Wen, and S. Cheng, "Probabilistic analysis of commutation failure in LCC-HVDC system considering the CFPREV and the initial fault voltage angle," IEEE Transactions on Power Delivery, pp. 1-1, 2019.

[15] H. Lee, G. T. Son, J. Yoo, and J. Park, "Effect of a SFCL on commutation failure in a HVDC system," IEEE Transactions on Applied Superconductivity, vol. 23, no. 3, pp. 5600 104-5 600 104, June 2013.

[16] L. Chen, H. Pan, C. Deng, F. Zheng, Z. Li, and F. Guo, "Study on the application of a flux-coupling-type superconducting fault current limiter for decreasing HVDC commutation failure," Canadian Journal of Electrical and Computer Engineering, vol. 38, no. 1, pp. 10-19, winter 2015. 